\title{
Threshold dynamics of a predator-prey model with age-structured prey
}

\author{
Yang $\mathrm{Lu}^{\prime}$ and Shengqiang $\mathrm{Liu}^{2 *}$ (1)
}

${ }^{\text {*Correspondence: sqliu@hit.edu.cn }}$ ${ }^{2}$ Department of Mathematics, Harbin Institute of Technology, Harbin, China

Full list of author information is available at the end of the article

\begin{abstract}
A predator-prey model, with aged structure in the prey population and the assumption that the predator hunts prey of all ages, is proposed and investigated. Using the uniform persistence theory for infinite dimensional dynamical systems, the global threshold dynamics of the model determined by the predator's net reproductive number $\Re_{P}$ are established: the predator-free equilibrium is globally stable if $\Re_{p}<1$, while the predator persists if $\Re_{p}>1$. Numerical simulations are given to illustrate the results.
\end{abstract}

MSC: Primary 92D25; secondary 34K20

Keywords: Predator-prey model; Age structured; Persistence; Delay integro-differential equations

\section{Introduction}

Predator-prey interactions are ubiquitous in the biological world, and they are one of the most important topics in ecology and continue to be of widespread interest today. Most existing studies on predator-prey models are focused on interacting species without age structure (see [1-4]). However, as the importance of age structure in populations has become more widely recognized, there is a rapidly growing literature dealing with various aspects of interacting populations with age structure [5-19].

In the above age-structured predator-prey population models, age structure is introduced into interactions of multi-species, and population models can quickly become remarkably complex $[7,13]$. Hence, it is understandable that many studies in the dynamics of age-structured predator-prey populations assumed that age structure was only employed in one species, either in predators or prey $[5,13-16,20]$. When considering the age structure among the prey population, we can assume that predation is dependent on the age of the prey. This allows us to include age-specific predation into the model and to reflect on different possible settings from biology. In [13], a general framework for age-structured predator-prey systems is introduced. However, Mohr et al. [13] assumed that only adult prey is involved in predation. In [11], Li et al. argued that the prey population should have an age structure and they assumed that the functional response is of predator-dependent type.

In this paper, we follow $[8,11,13,19,21,22]$ and propose a new predator-prey model with age-structured prey population. We assume that the predator hunts both the immature prey and the adult prey, and that the functional response of predators to prey is

(c) The Author(s) 2018. This article is distributed under the terms of the Creative Commons Attribution 4.0 International License (http://creativecommons.org/licenses/by/4.0/), which permits unrestricted use, distribution, and reproduction in any medium, provided you give appropriate credit to the original author(s) and the source, provide a link to the Creative Commons license, and indicate if changes were made. 
of Holling type II. Our primary aim of this paper is to obtain sharp criteria of the global threshold dynamics for the system.

The paper is organized as follows. In Sect. 2, we consider age-structured prey populations, define a threshold age, age-at-maturity, distinguish immature from adult individuals, and some assumption is introduced. In Sect. 3, we investigate the existence and stability of equilibria, and we find persistence. In the following section, we perform numerical simulations to verify our analytical results. At the end of the paper, we give a summary of the results.

\section{The model}

Throughout this paper, the indices 1 and 2 indicate variables and parameters related to larval and adult individuals, respectively.

- Let $P(t)$ denote the total number of the predator at time $t$. Assume that the predator population is governed by the Lotka-Volterra equation. $c(a)$ is the conversion efficiency of ingested prey into new predator individuals, and $m(a)>0$ is the per capita capture rate of prey by a searching predator, $h(a)>0$ is the handling (digestion) time per unit biomass consumed. In the absence of prey, the predator population, $P(t)$, decreases exponentially with rate $\mu_{P}>0$.

- Let $u(t, a)$ denote the prey population density of individuals of age $a$ at time $t$. Biological interpretation suggests that $\lim _{a \rightarrow+\infty} u(t, a)=0$, and we introduce a threshold age, $\tau>0$, to distinguish immature individuals $(a<\tau)$ from adult ones $(a \geq \tau)$. Thus, we distinguish immature prey, $u(t, a)=u_{1}(t, a), a \in[0, \tau)$, from adult prey, $u(t, a)=u_{2}(t, a), a \in[\tau,+\infty)$. The transition from the immature class to the adult one occurs at age $\tau>0$, the age-at-maturity of the prey. The total number of prey, $U(t)$, is given by

$$
U(t)=\int_{0}^{+\infty} u(t, a) d a=\int_{0}^{\tau} u_{1}(t, a) d a+\int_{\tau}^{+\infty} u_{2}(t, a) d a=U_{1}(t)+U_{2}(t) .
$$

$\mu:[0,+\infty) \rightarrow[0,+\infty)$ and $\beta:[0,+\infty) \rightarrow[0,+\infty)$ denote the age-dependent mortality and fertility rate of the prey, respectively. Here $\beta(\cdot) \in L_{+}^{\infty}((0,+\infty), \mathbb{R})$ clearly describes the effects of the age on the fertility.

Taking all the above into account, we have to study the following model:

$$
\left\{\begin{array}{l}
\frac{\partial u(t, a)}{\partial a}+\frac{\partial u(t, a)}{\partial t}=-\mu(a) u(t, a)-\frac{m(a) u(t, a)}{1+h(a) m(a) U(t)} P(t) \\
\frac{d P(t)}{d t}=\int_{0}^{\infty} \frac{c(a) m(a) u(t, a)}{1+h(a) m(a) U(t)} P(t) d a-\mu_{P} P(t), \\
u(0, \cdot)=u_{0} \in L^{1}((0,+\infty), \mathbb{R}), \quad P(0)=P_{0} \\
u(t, 0)=f(U(t)) \int_{0}^{+\infty} \beta(a) u(t, a) d a \\
u(t, a) \rightarrow 0 \quad \text { when } a \rightarrow+\infty
\end{array}\right.
$$

The number of newborns at time $t$ is $u(t, 0)<B^{+}$for all $t \geq 0, B^{+}$is a positive constant independent of age, the continuous function $u_{0}:[0,+\infty) \rightarrow[0,+\infty)$ provides the initial age distribution. The coefficient $f(U(t))$ measures the effects of the predation on the fertility of the prey, which is dependent on the total number of the prey, and we assume that the 
prey eggs will not be consumed by predators (see [11]), [18]):

$$
\begin{aligned}
& f(0)=0, \quad f(U(t)) \geq 0, \quad \forall U(t) \geq 0 ; \\
& f^{\prime}(U(t))>0, \quad \forall U(t) \geq 0, \quad \lim _{U(t) \rightarrow+\infty} f(U(t))=\iota ;
\end{aligned}
$$

$\exists U_{2}^{*}>0 \quad$ such that $\exp \left(-\mu_{1} \tau\right) \beta f\left(U_{2}^{*}\right)=\mu_{2}$.

In this paper, we assume that immature individuals are not fertile, so that $f(U(t))=$ $f\left(U_{2}(t)\right)$, then $u(t, 0)=f\left(U_{2}(t)\right) \beta U_{2}(t):=b\left(U_{2}(t)\right)$, it is clear that $b\left(U_{2}\right)$ is increasing, by (2.2), we get $\exp \left(-\mu_{1} \tau\right) b\left(U_{2}^{*}\right)=\mu_{2} U_{2}^{*}$. Note that (2.2) implies

$$
b^{\prime}(0) \exp \left(-\mu_{1} \tau\right)=\mu_{2} .
$$

For the age-structured prey population, we choose $\beta(a), m(a), \mu(a), c(a)$ and $h(a)$ in the form that these functions are constant for $a<\tau$ and for $a \geq \tau$, respectively. That is,

$$
\begin{aligned}
& \beta(a)=\left\{\begin{array}{ll}
0 & \text { for } a<\tau, \\
\beta & \text { for } a \geq \tau .
\end{array} \quad m(a)= \begin{cases}m_{1} & \text { for } a<\tau, \\
m_{2} & \text { for } a \geq \tau,\end{cases} \right. \\
& \mu(a)=\left\{\begin{array}{ll}
\mu_{1} & \text { for } a<\tau, \\
\mu_{2} & \text { for } a \geq \tau .
\end{array} \quad c(a)= \begin{cases}c_{1} & \text { for } a<\tau, \\
c_{2} & \text { for } a \geq \tau,\end{cases} \right. \\
& h(a)= \begin{cases}h_{1} & \text { for } a<\tau, \\
h_{2} & \text { for } a \geq \tau .\end{cases}
\end{aligned}
$$

We set up a modified Lotka-Sharpe model (see [23]) for $u_{1}(t, a), a<\tau$ :

$$
\begin{aligned}
& \frac{\partial u_{1}(t, a)}{\partial a}+\frac{\partial u_{1}(t, a)}{\partial t}=-\mu_{1} u_{1}(t, a)-\frac{m_{1} u_{1}(t, a)}{1+h_{1} m_{1} U(t)} P(t), \\
& \begin{aligned}
u_{1}(t, 0) & =f\left(U_{2}(t)\right) \int_{0}^{\infty} \beta(a) u(t, a) d a \\
& =b\left(U_{2}(t)\right), \\
u_{1}(0, a) & =u_{1}^{0}(a),
\end{aligned}
\end{aligned}
$$

with $u_{1}^{0}(a) \geq 0$ for all $a \in[0, \tau)$. Assuming that no individual dies at the very moment when it becomes adult, $u_{2}(t, \tau)=u_{1}(t, \tau)$, and that $\lim _{a \rightarrow+\infty} u_{2}(t, a)=0$, we have a similar system for $u_{2}(t, a)$ with initial age distribution $u_{2}^{0}(a) \geq 0$ for all $a>\tau$.

The total number of immature individuals satisfies

$$
\begin{aligned}
U_{1}^{\prime}(t) & =\int_{0}^{\tau} \frac{\partial u_{1}(t, a)}{\partial t} d a \\
& =-\int_{0}^{\tau}\left[\frac{\partial u_{1}(t, a)}{\partial a}+\mu_{1} u_{1}(t, a)+\frac{m_{1} u_{1}(t, a)}{1+h_{1} m_{1} U(t)} P(t)\right] d a \\
& =u_{1}(t, 0)-u_{1}(t, \tau)-\mu_{1} U_{1}(t)-\frac{m_{1} U_{1}(t)}{1+h_{1} m_{1} U(t)} P(t)
\end{aligned}
$$


and for the adult population we have

$$
\begin{aligned}
U_{2}^{\prime}(t) & =\int_{\tau}^{+\infty} \frac{\partial u_{2}(t, a)}{\partial t} d a \\
& =-\int_{\tau}^{+\infty}\left[\frac{\partial u_{2}(t, a)}{\partial a}+\mu_{2} u_{2}(t, a)+\frac{m_{2} u_{2}(t, a)}{1+h_{2} m_{2} U(t)} P(t)\right] d a \\
& =u_{2}(t, \tau)-\mu_{2} U_{2}(t)-\frac{m_{2} U_{2}(t)}{1+h_{2} m_{2} U(t)} P(t) \\
& =u_{1}(t, \tau)-\mu_{2} U_{2}(t)-\frac{m_{2} U_{2}(t)}{1+h_{2} m_{2} U(t)} P(t) .
\end{aligned}
$$

With the method of characteristics (see [24]) one finds the explicit solution of (2.4),

$$
u_{1}(t, a)= \begin{cases}u_{1}^{0}(a-t) e^{-\int_{0}^{t}\left(\mu_{1}+\frac{m_{1} P(s)}{1+h_{1} m_{1} U(s)}\right) d s}, & a>t, \\ b\left(U_{2}(t-a)\right) e^{-\int_{0}^{a}\left(\mu_{1}+\frac{m_{1} P(t-a+s)}{1+h_{1} m_{1} U(t-a+s)}\right) d s,} & a \leq t .\end{cases}
$$

When $t<\tau$, we get

$$
u_{1}(t, \tau)=u_{1}^{0}(\tau-t) e^{-\mu_{1} t-\int_{0}^{t} \frac{m_{1} P(s)}{1+h_{1} m_{1} U(s)} d s},
$$

and for $t \geq \tau$,

$$
u_{1}(t, \tau)=b\left(U_{2}(t-\tau)\right) e^{\left.-\mu_{1} \tau-\int_{0}^{\tau} \frac{m_{1} P(t-\tau+s)}{1+h_{1} m_{1} U(t-\tau+s)}\right) d s} .
$$

From the above derivation, for $t<\tau$, our age-structured prey models are as follows:

$$
\left\{\begin{aligned}
U_{1}^{\prime}(t)= & b\left(U_{2}(t)\right)-\mu_{1} U_{1}(t) \\
& -\frac{m_{1} U_{1}(t)}{1+h_{1} m_{1} U(t)} P(t)-u_{1}^{0}(\tau-t) e^{-\mu_{1} t-\int_{0}^{t} \frac{m_{1} P(s)}{1+h_{1} m_{1} U(s)} d s}, \\
U_{2}^{\prime}(t)= & u_{1}^{0}(\tau-t) e^{-\mu_{1} t-\int_{0}^{t} \frac{m_{1} P(s)}{1+h_{1} m_{1} U(s)} d s}-\mu_{2} U_{2}(t)-\frac{m_{2} U_{2}(t)}{1+h_{2} m_{2} U(t)} P(t), \\
\frac{d P(t)}{d t}= & c_{1} \cdot \frac{m_{1} U_{1}(t) P(t)}{1+h_{1} m_{1} U(t)}+c_{2} \cdot \frac{m_{2} U_{2}(t) P(t)}{1+h_{2} m_{2} U(t)}-\mu_{P} P(t) .
\end{aligned}\right.
$$

For $t \geq \tau$ we have

$$
\left\{\begin{aligned}
U_{1}^{\prime}(t)= & b\left(U_{2}(t)\right)-\mu_{1} U_{1}(t)-\frac{m_{1} U_{1}(t)}{1+h_{1} m_{1} U(t)} P(t) \\
& -b\left(U_{2}(t-\tau)\right) e^{-\mu_{1} \tau-\int_{0}^{\tau} \frac{m_{1} P(t-\tau+s)}{1+h_{1} m_{1} U(t-\tau+s)} d s}, \\
U_{2}^{\prime}(t)= & b\left(U_{2}(t-\tau)\right) e^{-\mu_{1} \tau-\int_{0}^{\tau} \frac{m_{1} P(t-\tau+s)}{1+h_{1} m_{1} U(t-\tau+s)} d s} \\
& -\mu_{2} U_{2}(t)-\frac{m_{2} U_{2}(t)}{1+h_{2} m_{2} U(t)} P(t), \\
\frac{d P(t)}{d t}= & c_{1} \cdot \frac{m_{1} U_{1}(t)}{1+h_{1} m_{1} U(t)} P(t)+c_{2} \cdot \frac{m_{2} U_{2}(t)}{1+h_{2} m_{2} U(t)} P(t)-\mu_{P} P(t) .
\end{aligned}\right.
$$

The meaning of all parameters can be found in Table 1 . 
Table 1 Variables and parameters used in the model

\begin{tabular}{ll}
\hline Symbol & Definition \\
\hline$U_{1}(t)$ & number of juvenile prey at time $t$ \\
$U_{2}(t)$ & number of mature prey at time $t$ \\
$P(t)$ & number of the predator at time $t$ \\
$u_{1}(t, a)$ & density of the juvenile prey at time $t$ of age $a$ \\
$u_{2}(t, a)$ & density of the mature prey at time $t$ of age $a$ \\
$\mu_{2}$ & per capita mortality rate of mature prey \\
$\mu_{1}$ & per capita mortality rate of juvenile prey \\
$\mu_{P}$ & per capita mortality rate for the predator \\
$c_{1}$ & prey juvenile biomass encounter rate \\
$c_{2}$ & prey adult biomass encounter rate \\
$\beta$ & the age-specific fertility rate or birth rate \\
$h(a)$ & the handling (digestion) time per unit biomass consumed \\
$m_{1}$ & the per capita capture rate of juvenile prey by a searching predator \\
$m_{2}$ & the per capita capture rate of mature prey by a searching predator \\
$\tau$ & maturation time of juvenile prey \\
$u(t, 0)$ & egg laying rate of mature prey \\
$\theta$ & maximum per capita female egg release \\
$f\left(U_{2}\right)$ & the effects of the predation on the fertility of mature prey: $\left(\theta U_{2}\right) /\left(1+\theta U_{2}\right)$ \\
\hline
\end{tabular}

\section{Mathematical analysis}

\subsection{Positivity and boundedness}

From the basic theory of delay differential equations (see, for example, [25]), the system (2.8) with the initial conditions

$$
\varphi(\theta)=\left(\varphi_{1}(\theta), \varphi_{2}(\theta), \varphi_{3}(\theta)\right), \quad \forall \theta \in[-\tau, 0], \varphi_{i}(\theta) \geq 0, \varphi_{i}(0)>0, i=1,2,3,
$$

and a unique solution $\left(U_{1}(t), U_{2}(t), P(t)\right)$ of system (2.8) is defined for all positive time provided that all solutions are bounded.

Throughout this section, we always assume that (2.2) holds.

Proposition 3.1 Suppose that (2.2) holds, then all the solutions of system (2.8) are nonnegative and bounded for all $t \geq 0$ on their respective initial intervals (3.1).

Proof We suppose that $u_{1}^{0}(a) \geq 0, a \geq 0$ is known, we take the solution of (2.7) as history function for (2.8) and we obtain nonnegative solutions if $u_{1}^{0}(a)$ is not known. Following [13, p. 100], we can obtain positivity of solutions.

It follows from [26, Theorem 5.2.1] that $U_{2}(t) \geq 0$ in its maximal interval of existence. We first show that the variables $U_{1}(t), U_{2}(t)$, and $P(t)$, with nonnegative initial data $U_{2}(0)>$ 0 , remain nonnegative as long as they exist. In fact, by the second equation of system (2.8), we have

$$
U_{2}(t)=U_{2}(0)\left(e^{-\mu_{2} t}+e^{\int_{0}^{t} \frac{m_{2} P(s)}{1+m_{2} h_{2} U(s)}}\right)+\int_{0}^{t} b\left(U_{2}(s-\tau)\right) \tilde{V} e^{\mu_{2}(s-t)} d s
$$

where

$$
\exp \left[-\int_{s-\tau}^{s}\left(\mu_{1}+\frac{m_{1} P(\eta)}{1+m_{1} h_{1} U(\eta)}\right) d \eta\right]:=\tilde{V} .
$$

Thus, $U_{2}(t)>0$ for all $t \geq 0$ holds, if it is not true, then there exists $\widetilde{t_{0}}>0$ such that $U_{2}\left(\widetilde{t_{0}}\right)=0$, let $t_{0}=\min \left\{\widetilde{t_{0}}: U_{2}\left(\widetilde{t_{0}}\right)=0\right\}$, so that $U_{2}\left(t_{0}\right)=0$ and $U_{2}(t)>0$ for all $t \in\left[0, t_{0}\right)$. 
On incorporation of the initial conditions, when $s<\tau$ for $s \in\left[0, t_{0}\right]$, we obtain

$$
U_{2}(0)\left(e^{-\mu_{2} t_{0}}+e^{\int_{0}^{t_{0}} \frac{m_{2} P(s)}{1+m_{2} h_{2} U(s)}}\right)+\int_{0}^{t} b\left(U_{2}(s-\tau)\right) \tilde{V} e^{\mu_{2}\left(s-t_{0}\right)} d s>0
$$

next if $s>\tau$ for $s \in\left[0, t_{0}\right]$, then, by assuming that $U_{2}(t)>0$ for all $t \in\left[0, t_{0}\right)$, we get

$$
U_{2}(0)\left(e^{-\mu_{2} t_{0}}+e^{\int_{0}^{t_{0}} \frac{m_{2} P(s)}{1+m_{2} h_{2} U(s)}}\right)+\int_{0}^{t} b\left(U_{2}(s-\tau)\right) \tilde{V} e^{\mu_{2}\left(s-t_{0}\right)} d s>0
$$

so that

$$
U_{2}(0)\left(e^{-\mu_{2} t_{0}}+e^{\int_{0}^{t_{0}} \frac{m_{2} P(s)}{1+m_{2} h_{2} U(s)}}\right)+\int_{0}^{t} b\left(U_{2}(s-\tau)\right) \tilde{V} e^{\mu_{2}\left(s-t_{0}\right)} d s>0
$$

By (3.2), this is a contradiction. Consequently, $U_{2}(t)>0$ for $t \geq 0$ holds.

The first equation of system (2.8) can be cast into an integral equation form, by differentiation, so that

$$
U_{1}(t)=\int_{t-\tau}^{t} b\left(U_{2}(\xi)\right) \exp \left(\int_{\xi}^{t}\left(-\mu_{1}-\frac{m_{1} P(\eta)}{1+m_{1} h_{1} U(\eta)}\right) d \eta\right) d \xi
$$

by (2.2) and $U_{2}(t)>0$ for all $t \geq 0$, we have $U_{1}(t)>0$ for all $t \geq 0$. Next, by the third equation of system (2.8),

$$
P(t)=P(0) e^{\int_{0}^{t}\left(c_{1} \cdot \frac{m_{1} U_{1}(s)}{1+m_{1} h_{1} U(s)}+c_{2} \cdot \frac{m_{2} U_{2}(s)}{1+m_{2} h_{2} U(s)}-\mu_{P}\right) d s},
$$

by (3.1), $U_{1}(t)>0, U_{2}(t)>0$ for all $t \geq 0$, thus $P(t)>0$ for all $t \geq 0$.

Next, we show that solutions remain bounded. Let

$$
V(t)=c_{0} U_{1}(t)+c_{0} U_{2}(t)+P(t)
$$

where $c_{0}=\max \left\{c_{1}, c_{2}\right\}$, calculating the derivative of $V(t)$ along trajectories of system (2.8), we obtain

$$
\begin{aligned}
V^{\prime}(t)= & c_{0} b\left(U_{2}(t)\right)-c_{0} \mu_{2} U_{2}(t)-c_{0} \mu_{1} U_{1}(t)-\mu_{P} P(t) \\
& -c_{0} \cdot \frac{m_{2} U_{2}(t) P(t)}{1+m_{2} h_{2} U(t)}-c_{0} \cdot \frac{m_{1} U_{1}(t) P(t)}{1+m_{1} h_{1} U(t)} \\
& +c_{1} \cdot \frac{m_{1} U_{1}(t) P(t)}{1+m_{1} h_{1} U(t)}+c_{2} \cdot \frac{m_{2} U_{2}(t) P(t)}{1+m_{2} h_{2} U(t)} \\
\leq & c_{0} b\left(U_{2}(t)\right)-c_{0} \mu_{2} U_{2}(t)-c_{0} \mu_{1} U_{1}(t)-\mu_{P} P(t) \\
& -c_{0} \cdot \frac{m_{2} U_{2}(t) P(t)}{1+m_{2} h_{2} U(t)}-c_{0} \cdot \frac{m_{1} U_{1}(t) P(t)}{1+m_{1} h_{1} U(t)} \\
& +c_{0} \cdot \frac{m_{1} U_{1}(t) P(t)}{1+m_{1} h_{1} U(t)}+c_{0} \cdot \frac{m_{2} U_{2}(t) P(t)}{1+m_{2} h_{2} U(t)} \\
\leq & c_{0} B^{+}-c_{0} \mu_{1} U_{1}(t)-c_{0} \mu_{2} U_{2}(t)-\mu_{P} P(t),
\end{aligned}
$$


for positive constant $\sigma\left(\sigma=\min \left\{\mu_{1}, \mu_{2}, \mu_{P}\right\}\right)$, it follows from (3.3) that

$$
V^{\prime}(t)+\sigma V(t) \leq c_{0} B^{+}
$$

and this yields

$$
\limsup _{t \rightarrow+\infty} V(t)<\frac{c_{0} B^{+}}{\sigma}
$$

where $u(t, 0)<B^{+}$for all $t \geq 0, B^{+}$is a positive constant independent of age, apparently, $u(t, 0)=b\left(U_{2}(t)\right)<B^{+}$for all $t \geq 0$. Then $U_{1}(t), U_{2}(t), P(t)$ are bounded. Consequently, the solution $\left(U_{1}(t), U_{2}(t), P(t)\right)$ of system (2.8) with initial condition (3.1) exists for all $t \geq 0$.

\subsection{Existence of the boundary equilibria}

The equilibrium $\left(\overline{U_{1}}, \overline{U_{2}}, \bar{P}\right)$ of system (2.8) satisfies the following system:

$$
\left\{\begin{array}{l}
b\left(\overline{U_{2}}\right)-\mu_{1} \overline{U_{1}}-\frac{m_{1} \overline{U_{1}}}{1+m_{1} h_{1} \bar{P}}-b\left(\overline{U_{2}}\right) e^{-\mu_{1} \tau-\int_{0}^{\tau} \frac{m_{1} \bar{P}}{1+m_{1} h_{1} \bar{U}} d s}=0, \\
b\left(\overline{U_{2}}\right) e^{-\mu_{1} \tau-\int_{0}^{\tau} \frac{m_{1} \bar{P}}{1+m_{1} h_{1} \bar{U}} d s}-\mu_{2} \overline{U_{2}}-\frac{m_{2} \overline{U_{2}}}{1+m_{2} h_{2} \bar{U}} \bar{P}=0, \\
c_{1} \cdot \frac{m_{1} \overline{U_{1}}}{1+m_{1} h_{1} \bar{U}} \bar{P}+c_{2} \cdot \frac{m_{2} \overline{U_{2}}}{1+m_{2} h_{2} \bar{U}} \bar{P}-\mu_{P} \bar{P}=0 .
\end{array}\right.
$$

It is easy to see that the equilibrium point $E_{0}=(0,0,0)$ always exists for all parameter values. If (2.2) holds, there is an equilibrium with $P=0$; in the predator-free equilibrium, the $U_{1}$ and $U_{2}$ components are $U_{1}^{*}$ and $U_{2}^{*}$ with $U_{2}^{*}>0$ from (2.2), which satisfy

$$
\exp \left(-\mu_{1} \tau\right) b\left(U_{2}^{*}\right)=\mu_{2} U_{2}^{*}, \quad U_{1}^{*}=\frac{b\left(U_{2}^{*}\right)\left(1-\exp \left(-\mu_{1} \tau\right)\right)}{\mu_{1}} .
$$

\subsection{Persistence and stability analysis}

In this section, we study the global stability of the predator-free equilibrium $E_{1}$ of (2.8). Our principal result in this section can be stated as follows.

Theorem 3.1 Let $\Re_{P}:=\frac{1}{\mu_{P}}\left\{\frac{c_{1} m_{1} U_{1}^{*}(t)}{1+h_{1} m_{1} U^{*}(t)}+\frac{c_{2} m_{2} U_{2}^{*}(t)}{1+h_{2} m_{2} U^{*}(t)}\right\}$, where $U^{*}(t)=U_{1}^{*}(t)+U_{2}^{*}(t)$. The predator-free equilibrium $E_{1}$ of $(2.8)$ is globally asymptotically stable if $\Re_{P}<1$.

Proof We use the variant of system that involves the first and second equations of system (2.8). From the second equation of system (2.8), and using positivity of solutions,

$$
\frac{d U_{2}(t)}{d t} \leq-\mu_{2} U_{2}(t)+\exp \left(-\mu_{1} \tau\right) b\left(U_{2}(t-\tau)\right)
$$

Since $b(\cdot)$ is increasing, we may use a comparison argument (for example see [26]) to conclude that $U_{2}(t)$ is bounded by the solution of the corresponding differential equation obtained from (3.7) by changing $\leq$ to $=$. Since $b(\cdot)$ is increasing, positive solutions of that differential equation approach $U_{2}^{*}$ (see [27]). Therefore,

$$
\limsup _{t \rightarrow \infty} U_{2}(t) \leq U_{2}^{*}
$$


and, from the first equation of system (2.8), we have

$$
\limsup _{t \rightarrow \infty} U_{1}(t) \leq \frac{b\left(U_{2}^{*}\right)\left(1-\exp \left\{-\mu_{1} \tau\right\}\right)}{\mu_{1}}=U_{1}^{*}
$$

Since $\Re_{P}<1$ holds, there exists a positive small constant $\epsilon$ such that

$$
\mu_{P}>\left\{\frac{c_{1} m_{1}\left(U_{1}^{*}+\epsilon\right)}{1+h_{1} m_{1}\left(U^{*}+\epsilon\right)}+\frac{c_{2} m_{2}\left(U_{2}^{*}+\epsilon\right)}{1+h_{2} m_{2}\left(U^{*}+\epsilon\right)}\right\} .
$$

With this $\epsilon, \exists T$, such that $U_{1}(t) \leq U_{1}^{*}+\epsilon$ for $t>T$. From the third equation of system (2.8) and for $t$ sufficiently large we obtain

$$
\frac{d P(t)}{d t} \leq \frac{c_{1} m_{1}\left(U_{1}^{*}+\epsilon\right) P(t)}{1+h_{1} m_{1}\left(U^{*}+\epsilon\right)}+\frac{c_{2} m_{2}\left(U_{2}^{*}+\epsilon\right) P(t)}{1+h_{2} m_{2}\left(U^{*}+\epsilon\right)}-\mu_{P} P(t) .
$$

We introduce the following auxiliary equation:

$$
\frac{d V(t)}{d t}=\frac{c_{1} m_{1}\left(U_{1}^{*}+\epsilon\right) V(t)}{1+h_{1} m_{1}\left(U^{*}+\epsilon\right)}+\frac{c_{2} m_{2}\left(U_{2}^{*}+\epsilon\right) V(t)}{1+h_{2} m_{2}\left(U^{*}+\epsilon\right)}-\mu_{P} V(t)
$$

with $V(0)=P(0)$. By the comparison theorem, we have

$$
P(t) \leq V(t), \quad t \geq 0
$$

Rearranging $\Re_{P}<1$, thus

$$
\left\{\frac{c_{1} m_{1}\left(U_{1}^{*}+\epsilon\right)}{1+h_{1} m_{1}\left(U^{*}+\epsilon\right)}+\frac{c_{2} m_{2}\left(U_{2}^{*}+\epsilon\right)}{1+h_{2} m_{2}\left(U^{*}+\epsilon\right)}\right\}<\mu_{P} .
$$

By [28, Lemma 2], this implies $\lim _{t \rightarrow+\infty} V(t)=0$. Combining Proposition 3.1 and (3.11), we therefore have

$$
\lim _{t \rightarrow+\infty} P(t)=0
$$

With the above analysis, we get $\lim _{t \rightarrow+\infty}\left(U_{1}(t), U_{2}(t), P(t)\right)=\left(U_{1}^{*}, U_{2}^{*}, 0\right)$. so that the predator-free equilibrium $E_{1}$ of (2.8) is globally asymptotically stable if $\Re_{P}<1$. This completes the proof of Theorem 3.1.

Theorem 3.2 Suppose (2.2)-(2.3) hold. If $\Re_{P}>1$, the predator $P$ is uniformly persists. Namely, there exists $\delta>0$, which is independent of the initial conditions, such that

$$
\liminf _{t \rightarrow \infty} P(t)>\delta
$$

Proof Next we apply [29, Theorem 1.3.2] to prove and establish population persistence. Let $C^{+}\left([-\tau, 0], R_{+}^{3}\right)$ denote the space of continuous functions mapping $[-\tau, 0]$ into $R_{+}^{3}$. Denote

$$
M:=C^{+}\left([-\tau, 0], R_{+}^{3}\right), \quad M^{0}:=\left\{\varphi \in M: \varphi_{i}(0)>0, i=1,2,3\right\},
$$


and

$$
\partial M^{0}=M \backslash M^{0}
$$

Clearly, $M^{0}$ is an open set relative to $M$. Define $T$ to be a continuous semiflow on $M$, i.e., for any $t \geq 0, T(t)$ is a $C^{0}$-semigroup on $M$ satisfying

$$
T(t): M^{0} \rightarrow M^{0}, \quad T(t): \partial M^{0} \rightarrow \partial M^{0}
$$

and

$$
T(t) \varphi(\theta)=\left(U_{1}(t+\theta), U_{2}(t+\theta), P(t+\theta)\right)
$$

where $\left(U_{1}(t), U_{2}(t), P(t)\right)$ is the solution of system (2.8) with initial conditions (3.1). By the definitions of $M^{0}$ and $\partial M^{0}$ and Theorem 3.1, it is easy to see that a constant $t_{0} \geq 0$ exists such that $T(t)$ is compact for all $t>t_{0} ; T(t)$ is point dissipative. Let $\omega(\varphi)$ be the omega limit set of the orbit

$$
\gamma^{+}(\varphi)=\{T(t) \varphi: \forall t \geq 0\}
$$

and define $M_{\partial}$ the particular invariant set, i.e.,

$$
M_{\partial}=\left\{\varphi \in \partial M^{0}: T(t) \varphi \in \partial M^{0}, \forall t \geq 0\right\} .
$$

From the proof of Proposition 3.1, we know that

$$
M_{\partial}=\left\{\varphi \in \partial M^{0}: \varphi_{2}=0 \cup \varphi_{3}=0\right\} .
$$

Therefore,

$$
\omega(\varphi)=\left\{(0,0,0),\left(U_{1}^{* *}, U_{2}^{* *}, 0\right)\right\}, \quad \forall \varphi \in M_{\partial} .
$$

By Theorem 3.1, we can see that the flow in $M_{\partial}$ is isolated and acyclic.

To complete the proof of Theorem 3.2, we now need to prove the following two claims.

Claim $1 W^{s}\left(E_{0}\right) \cap M^{0}=\emptyset$. Assume $W^{s}\left(E_{0}\right) \cap M^{0} \neq \emptyset$, i.e., there exists a positive solution $\left(U_{1}(t), U_{2}(t), P(t)\right)$ satisfying $\lim _{t \rightarrow+\infty}\left(U_{1}(t), U_{2}(t), P(t)\right)=(0,0,0)$. For sufficiently small positive constant $\eta$, there exists $T_{1}$ such that

$$
U_{1}(t)<\eta, \quad U_{2}(t)<\eta, \quad P(t)<\eta \quad \text { for all } t \geq T_{1} .
$$

From the second equation of system (2.8) and (2.2), this implies that

$$
\begin{aligned}
U_{2}^{\prime}(t) & >b\left(U_{2}(t-\tau)\right) e^{-\mu_{1} \tau-m_{1} \eta \tau}-\left(\mu_{2}+m_{2} \eta\right) U_{2}(t) \\
& =\beta f\left(U_{2}(t-\tau)\right) e^{-\mu_{1} \tau-m_{1} \eta \tau} U_{2}(t-\tau)-\left(\mu_{2}+m_{2} \eta\right) U_{2}(t),
\end{aligned}
$$


for all $t \geq T_{1}$, by (2.2)-(2.3), $\mu_{2}<\beta f\left(U_{2}(t-\tau)\right) e^{-\mu_{1} \tau}$, for sufficiently small positive constant $\eta$, then

$$
\beta f\left(U_{2}(t-\tau)\right) e^{-\mu_{1} \tau-m_{1} \eta \tau}>\mu_{2}+m_{2} \eta
$$

Consider the equation

$$
\left\{\begin{array}{l}
\psi^{\prime}(t)=\beta f(\psi(t-\tau)) e^{-\mu_{U, 1} \tau-m_{1} \eta \tau} \psi(t-\tau)-\left(\mu_{U_{2}}+m_{2} \eta\right) \psi(t), \quad t \geq T_{1} \\
\psi(t)=U_{2}(t), \quad t \in\left[T_{1}, T_{1}+\tau\right] .
\end{array}\right.
$$

By (3.13) the comparison theorem, we have $U_{2}(t) \geq \psi(t)$ for all $t>T_{1}$. On the other hand, using [27, Theorem 4.9.1], we have $\lim _{t \rightarrow+\infty} \psi(t)=\psi^{*}$ for all solutions to system (3.14), where $\psi^{*}>\eta$ is the unique positive equilibrium of system (3.14). Hence we obtain $\lim \sup _{t \rightarrow+\infty} U_{2}(t) \geq \psi^{*}>\eta$, contradicting $P(t)<\eta$ as $t \geq T_{1}$. We therefore conclude that $W^{s}\left(E_{0}\right) \cap M^{0}=\emptyset$.

Claim 2 Now we verify $W^{s}\left(E_{1}\right) \cap M^{0}=\emptyset$. Assume this is not true, i.e., $W^{s}\left(E_{1}\right) \cap M^{0} \neq \emptyset$, then there exists a positive solution $\left(U_{1}(t), U_{2}(t), P(t)\right)$ of system $(2.8)$ such that $\lim _{t \rightarrow+\infty}\left(U_{1}(t)\right.$, $\left.U_{2}(t), P(t)\right)=\left(U_{1}^{*}, U_{2}^{*}, 0\right)$, where $U^{*}=U_{1}^{*}+U_{2}^{*}$. For the same value of $\eta$ as that in Claim 1 , there exists a positive constant $T_{2} \geq T_{1}$ such that

$$
U_{1}(t)>U_{1}^{*}-\eta, \quad U_{2}^{*}-\eta<U_{2}(t)<U_{2}^{*}+\eta, \quad P(t)<\eta, \quad \text { for all } t \geq T_{2} .
$$

From the third equation of system (2.8) we have

$$
P^{\prime}(t)>\frac{c_{1} m_{1}\left(U_{1}^{*}-\eta\right)}{1+m_{1} h_{1}\left(U^{*}-\eta\right)} P(t)+\frac{c_{2} m_{2}\left(U_{2}^{*}-\eta\right)}{1+m_{2} h_{2}\left(U^{*}-\eta\right)} P(t)-\mu_{P} P(t)
$$

for all $t>T_{2}+\tau$. Integrating both sides of (3.15) yields

$$
P(t)>p_{0} \exp \left\{\int_{0}^{t}\left[\frac{c_{1} m_{1}\left(U_{1}^{*}-\eta\right)}{1+m_{1} h_{1}\left(U^{*}-\eta\right)}+\frac{c_{2} m_{2}\left(U_{2}^{*}-\eta\right)}{1+m_{2} h_{2}\left(U^{*}-\eta\right)}-\mu_{P}\right] d s\right\},
$$

by $\Re_{P}>1$, we get

$$
\frac{c_{1} m_{1}\left(U_{1}^{*}-\eta\right)}{1+m_{1} h_{1}\left(U^{*}-\eta\right)}+\frac{c_{2} m_{2}\left(U_{2}^{*}-\eta\right)}{1+m_{2} h_{2}\left(U^{*}-\eta\right)}>\mu_{P}
$$

which contradicts with $P(t)<\eta$ as $t \geq T_{2}+\tau$. So that we conclude that $W^{s}\left(E_{1}\right) \cap M^{0}=\emptyset$.

The above two claims show that $E_{0}, E_{1}$ are uniform weak repellers for $M^{0}$ in the sense that

$$
\limsup _{t \rightarrow+\infty}\left\|T(t) \varphi-E_{i}\right\| \geq \eta, \quad \forall \varphi \in M^{0}, i=0,1
$$

with the maximum norm $\|\cdot\|$. Thus, from [29, Theorem 1.3.2], we find that there exists a constant $\delta>0$ such that

$$
\liminf _{t \rightarrow+\infty} P(t)>\delta
$$


Figure 1 Density of the predator $P$ of system (2.8) as $\Re_{P}=1.0901>1$. The other parameters are as follows: $\mu_{1}=0.092, \mu_{2}=0.23, \mu_{P}=0.9, \beta=0.99, h_{1}=0.009$, $m_{1}=0.09, h_{2}=0.0095, m_{2}=0.15, c_{1}=1, c_{2}=1$, $\tau=12$

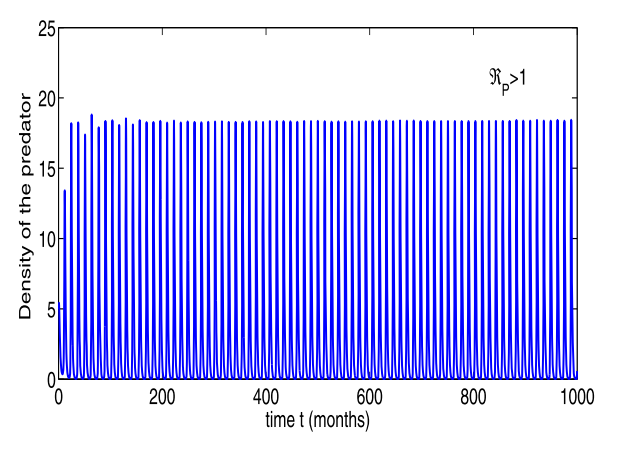

uniformly for all solutions of system (2.8), which implies that the system (2.8) is uniformly persistent if $\Re_{P}>1$ holds. This completes the proof of Theorem 3.2.

\section{Numerical simulations}

In this section we conduct numerical simulations to illustrate our analytical results. Parameter values are taken from Table 1. In all of the simulations we measure the time in months. We choose the effects of the predation on the fertility of prey to be $f\left(U_{2}(t)\right)=$ $\frac{\theta U_{2}(t)}{1+\theta U_{2}(t)}$, we choose parameters $\theta=2, U_{1}(0)=5, U_{2}(0)=5, P(0)=5$, other parameters values are listed in caption of each figure, and system (2.8) becomes

$$
\left\{\begin{aligned}
U_{1}^{\prime}(t)= & \frac{\beta \theta\left(U_{2}(t)\right)^{2}}{1+\theta U_{2}(t)}-\mu_{1} U_{1}(t)-\frac{m_{1} U_{1}(t)}{1+h_{1} m_{1} U(t)} P(t) \\
& -\frac{\beta \theta\left(U_{2}(t-\tau)\right)^{2}}{1+\theta U_{2}(t-\tau)} e^{-\mu_{1} \tau-\int_{0}^{\tau} \frac{m_{1} P(t-\tau+s)}{1+h_{1} m_{1} U(t-\tau+s)} d s}, \\
U_{2}^{\prime}(t)= & \frac{\beta \theta\left(U_{2}(t-\tau)\right)^{2}}{1+\theta U_{2}(t-\tau)} e^{-\mu_{1} \tau-\int_{0}^{\tau} \frac{m_{1} P(t-\tau+s)}{1+h_{1} m_{1} U(t-\tau+s)} d s} \\
& -\mu_{2} U_{2}(t)-\frac{m_{2} U_{2}(t)}{1+h_{2} m_{2} U(t)} P(t), \\
\frac{d P(t)}{d t}= & c_{1} \cdot \frac{m_{1} U_{1}(t)}{1+h_{1} m_{1} U(t)} P(t)+c_{2} \cdot \frac{m_{2} U_{2}(t)}{1+h_{2} m_{2} U(t)} P(t)-\mu_{P} P(t) .
\end{aligned}\right.
$$

The boundary equilibria are

$$
E_{0}=(0,0,0), \quad E_{1}=\left(\frac{\mu_{2}^{2} e^{\mu_{1} \tau}\left(e^{\mu_{1} \tau}-1\right)}{\mu_{1} \theta\left(\beta-\mu_{2} e^{\mu_{1} \tau}\right)}, \frac{\mu_{2} e^{\mu_{1} \tau}}{\theta\left(\beta-\mu_{2} e^{\mu_{1} \tau}\right)}, 0\right) .
$$

The predator's net reproductive number $\Re_{P}$ is

$$
\Re_{P}:=\frac{1}{\mu_{P}}\left\{Q_{1}+Q_{2}\right\},
$$

where

$$
\begin{aligned}
Q_{1} & =\frac{c_{1} m_{1} \mu_{2}^{2} e^{\mu_{1} \tau}\left(e^{\mu_{1} \tau}-1\right)}{\mu_{1} \theta\left(\beta-\mu_{2} e^{\mu_{1} \tau}\right)+h_{1} m_{1} \mu_{2}^{2} e^{\mu_{1} \tau}\left(e^{\mu_{1} \tau}-1\right)}, \\
Q_{2} & =\frac{c_{2} m_{2} \mu_{2} e^{\mu_{1} \tau}}{\theta\left(\beta-\mu_{2} e^{\mu_{1} \tau}\right)+h_{2} m_{2} \mu_{2} e^{\mu_{1} \tau}} .
\end{aligned}
$$

Figure 1 shows that the predator $P$ is uniformly persists if $\Re_{P}>1$ (see Theorem 3.2). 

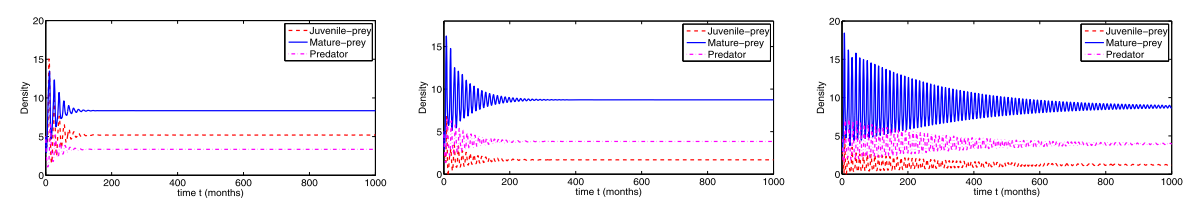

Figure 2 Solutions to the system (2.8) with different capture rates. (a): $m_{1}=0.08, m_{2}=0.15 ;(\mathbf{b}): m_{1}=0.13$, $m_{2}=0.15 ;(\mathbf{c}): m_{1}=0.147, m_{2}=0.15$. The other parameters are as follows: $\mu_{1}=0.005, \mu_{2}=0.13, \mu_{P}=0.55$, $\beta=0.85, m_{1} h_{1}=m_{2} h_{2}=0.0001, c_{1}=0.12, c_{2}=0.40, \tau=6$
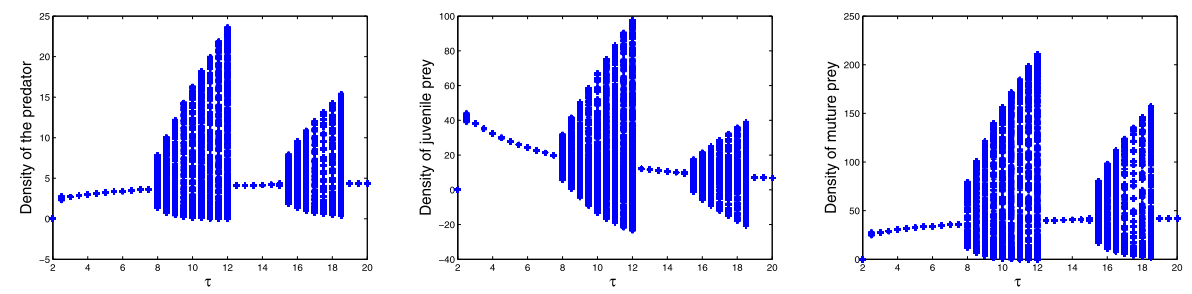

Figure 3 The ultimate oscillation interval of the solution to system (2.8) when $\tau$ increases from 0 to 20 , here $t \in[10,2000] . \mu_{1}=0.005 ; \mu_{2}=0.13 ; \mu_{P}=0.85 ; m_{1}=0.08 ; c_{1}=0.11 ; c_{2}=0.125 ; m_{1} h_{1}=m_{2} h_{2}=0.0001 ;$ $m_{2}=0.15 ; \beta=0.85$

Figure 2 shows that if the predator hunts juvenile prey increasingly large, the size of the predator and mature prey to be expanded, this capture strategy will accelerate the extinction of juvenile prey.

From Fig. 3, we see that if $\tau \in(0,8) \operatorname{or}(12,15.5)$, approximately, the vertical amplitudes of $P(t), U_{1}(t)$ and $U_{2}(t)$ are as small as a point, suggesting that they are asymptotically stable; if $\tau$ increases in the interval $[8,12]$ or $[15.5,18.5]$, approximately, the vertical amplitudes of $P(t), U_{1}(t)$ and $U_{2}(t)$ will become larger and larger, showing that they become more and more unstable.

\section{Summary and discussion}

In this paper, we study a predator-prey system with stage structured on the prey. The predator hunts both the immature prey and the adult prey. We have developed a rigorous analysis of the model by applying the comparison theory of differential equations and uniform persistence theory. Global dynamics of the model are obtained and threshold dynamics determined by the predator's net reproductive number $\Re_{P}$ are established: the predators go extinct if $\Re_{P}<1$; and predators persist if $\Re_{P}>1$. Theorem 3.1 shows that the predator-free equilibrium $E_{1}$ of (2.8) is globally asymptotically stable if $\Re_{P}<1$. That the predator $P$ is uniformly persistent is also obtained in Theorem 3.2.

First, we have constructed the predator's net reproductive number $\Re_{P}$, and by applying the comparison theory of differential equations, we get the predator-free equilibrium $E_{1}$ of (2.8) is globally asymptotically stable if $\Re_{P}<1$ (see Theorem 3.1).

Second, by applying the uniform persistence theory, the predator $P$ is uniformly persistent is also obtained in Theorem 3.2 (see Fig. 1).

Besides the above systematic theoretical results for model (2.8), we also perform careful numerical simulations to support the theoretical results. The prey have stage structure and the highlights of this paper are the effects by delay $\tau$. It is shown that of the immature prey $\tau$ 
largely determines stability of the immature prey and the predator, in addition $\tau$ increases from 8 to $12 / 15.5$ to 18.5 , and the predator may lose its stability and becomes increasingly unstable by enlarging the amplitude of the oscillation interval (see Fig. 3). Biologically, this means that a shorter immature prey maturation period is helpful to stabilize the system.

In this paper, the stability of the predator-prey coexistence equilibrium remains unclear, which we leave as our future work.

\begin{abstract}
Acknowledgements
The authors would like to express their gratitude to the referees and the editor for their constructive, scientific thorough suggestions and comments on the manuscript. SL is supported by the NNSF of China (No. 11471089 and No. 11771374).
\end{abstract}

\title{
Competing interests
}

The authors declare that they have no competing interests.

\section{Authors' contributions}

$Y L$ and SL performed the mathematical analysis of the model, wrote and typeset the manuscript, and conducted the numerical simulations and discussions. SL formulated the model. Both authors read and approved the final manuscript.

\section{Author details}

${ }^{1}$ College of Mathematics and Statistics, Northeast Petroleum University, Daqing, China. ${ }^{2}$ Department of Mathematics, Harbin Institute of Technology, Harbin, China.

\section{Publisher's Note}

Springer Nature remains neutral with regard to jurisdictional claims in published maps and institutional affiliations.

Received: 5 December 2017 Accepted: 24 April 2018 Published online: 05 May 2018

\section{References}

1. Xiao, Y., Chen, L.: Modeling and analysis of a predator-prey model with disease in the prey. Math. Biosci. 171, 59-82 (2001)

2. Liu, S., Chen, L., Liu, Z.: Extionction and permanence in nonautonomous competitive system with stage structure. J. Math. Anal. Appl. 274, 667-684 (2002)

3. Fan, M., Kuang, Y.: Dynamics of a non-autonomous predator-prey system with the Beddington-Deangelis functional response. J. Math. Anal. Appl. 295, 15-39 (2004)

4. Rui, X., Chaplain, M.A.J., Davidson, F.A.: Permanence and periodicity of a delayed ratio-dependent predator-prey model with stage-structure. J. Math. Anal. Appl. 303, 602-621 (2005)

5. Lu, Y., Pawelek, P.A., Liu, S.: A stage-structured predator-prey model with predation over juvenile prey. Appl. Math. Comput. 297, 115-130 (2017)

6. Liu, S., Beretta, E.: A stage-structured predator-prey model of Beddington-DeAngelis type. SIAM J. Appl. Math. 66 1101-1129 (2006)

7. Gourley, S., Lou, Y.: A mathematical model for the spatial spread and biocontrol of the astan longhorned beetle. SIAM J. Appl. Math. 74, 864-884 (2014)

8. Fang, J., Gourley, S., Lou, Y.: Stage-structured models of intra- and inter-specific competition within age classes. J. Differ. Equ. 260, 1918-1953 (2016)

9. Browne, C., Pilyugin, S.: Global analysis of age-structured within-host virus model. Discrete Contin. Dyn. Syst., Ser. B 18, 1999-2017 (2013)

10. Van Den Driessche, P., Wang, L., Zou, X.: Modeling diseases with latency and relapse. Math. Biosci. Eng. 4, 205-219 (2007)

11. Li, J.: Dynamics of age-structured predator-prey population models. J. Math. Anal. Appl. 152, 399-415 (1990)

12. Delgado, M., Becerra, M., Suarez, A.: Analysis of an age-structured predator-prey model with disease in the prey. Nonlinear Anal., Real World Appl. 7, 853-871 (2006)

13. Mohr, M., Barbarossa, M.V., Kuttler, C.: Predator-prey interactions, age structures and delay equations. Math. Model. Nat. Phenom. 9, 92-107 (2014)

14. Liu, Z., Magal, P., Ruan, S.: Predator-prey interactions, age structures and delay equations. Discrete Contin. Dyn. Syst., Ser. B 21, 537-555 (2016)

15. Tang, H., Liu, Z.: Hopf bifurcation for a predator-prey model with age structure. Appl. Math. Model. 40, 726-737 (2016)

16. Liu, Z., Li, N.: Stability and bifurcation in a predator-prey model with age structure and delays. J. Nonlinear Sci. 25 , 937-957 (2015)

17. Bocharov, G., Hadeler, K.P.: Structured population models, conservation laws, and delay equations. J. Differ. Equ. 168, 212-237 (2000)

18. Fister, K., Lenhart, S.: Optimal harvesting in an age-structured predator-prey model. Appl. Math. Optim. 54, 1-15 (2006)

19. Liu, S., Xie, X., Tang, J.: Competing population model with nonlinear intraspecific regulation and maturation delays. Int. J. Biomath. 5, 1260007-1-1260007-22 (2012)

20. Cushing, J., Saleem, M.: A predator-prey model with age-structure. J. Math. Biol. 14, 231-250 (1982) 
21. Li, Y., Wang, J., Sun, B., Tang, J., Xie, X., Pang, S.: Modeling and analysis of the secondary routine dose against measles in China. Adv. Differ. Equ. 2017, 89 (2017)

22. Qiu, L., Yao, F., Zhong, X.: Stability analysis of networked control systems with random time delays and packet dropouts modeled by Markov chains. J. Appl. Math. 2013, 715072 (2013)

23. Sharpe, F.R., Lotka, A.J.: A problem in age distribution. Philos. Mag. Ser. 6 21, 435-438 (1911)

24. Evans, L.C.: Partial Differential Equations. AMS, Providence (1998)

25. Hale, J.K., Verduyn Lunel, S.M.: Introduction to Functional Differential Equations. Springer, New York (1993)

26. Smith, H.L.: Monotone Dynamical Systems: An Introduction to the Theory of Competitive and Cooperative Systems. Amer. Math. Soc., Providence (1995)

27. Kuang, Y.: Delay Differential Equations with Applications in Population Dynamics. Academic Press, New York (1993)

28. Liu, S., Chen, L., Luo, G., Jiang, L.: Asymptotic behavior of competitive Lotka-Volterra system with stage structure. J. Math. Anal. Appl. 271, 124-138 (2002)

29. Zhao, X.Q.: Dynamical System in Population Biology. Springer, New York (2003)

\section{Submit your manuscript to a SpringerOpen ${ }^{\circ}$} journal and benefit from:

- Convenient online submission

- Rigorous peer review

- Open access: articles freely available online

- High visibility within the field

- Retaining the copyright to your article

Submit your next manuscript at $\boldsymbol{~ s p r i n g e r o p e n . c o m ~}$ 